\title{
Gestión curricular: la experiencia de la Licenciatura en Enfermería de la Universidad de Costa Rica ${ }^{1}$
}

Ana Guzman Aguilar ${ }^{2}$

Institución: Universidad de Costa Rica

\section{COMO CITAR}

Guzmán, A. (abril, 2013). Gestión curricular: la experiencia de la Licenciatura en Enfermería de la Universidad de Costa Rica.. Rev. Actual en Costa Rica, 24, 1-14. Recuperado de:: <http://www.revenf.ucr.ac.cr/gestion.pdf> ISSN 1409-4568

\section{Resumen}

Se presenta en este documento los resultados del tercer objetivo de la investigación titulada Análisis del Plan de Estudios de la Licenciatura en Enfermeria de la Universidad de Costa Rica. Este objetivo redunda en la estructura de la gestión curricular del plan en mención. Participaron en este proceso todos los docentes de la Escuela de Enfermería, docentes de servicio, estudiantes de quinto año de la Licenciatura, egresados y empleadores en el año 2011-2012. Se desarrolló un enfoque mixto, con un diseño paralelo. Para la recolección de información, se utilizaron técnicas orales y documentales, instrumentos como cuestionarios y entrevistas semiestructuradas a docentes, estudiantes y empleadores, así como la revisión de los documentos del diseño curricular del plan de estudios, Las variables analizadas para el tercer objetivo fueron suficiencia e idoneidad del personal docente, suficiencia en la infraestructura y suficiencia e idoneidad de los campos clínicos. Se destacó entre los resultados que la gestión curricular del plan de estudios fue valorada en términos generales de manera satisfactoria en todos sus rubros; lo que conlleva a impulsar el mejoramiento y la calidad en la formación de los y las profesionales en Enfermería. Se concluye que existe suficiencia e idoneidad de los y las docentes de la escuela de Enfermería y el programa de desarrollo del personal docente es una acción positiva en este sentido. La Infraestructura de la planta física aun no cumple con las expectativas de docentes y estudiantes. Por último, la restricción en el número de estudiantes que se aceptan por campo clínico según el actual Convenio CCSS-UCR y la cantidad de estudiantes que admite la unidad académica anualmente hace que sean insuficientes por lo que al incorporar la simulación clínica el estudiantado logra aprovechar al máximo las experiencias de aprendizaje en los servicios de salud.

Palabras clave: Curriculum, Enfermería, Gestión

\footnotetext{
${ }^{1}$ Fecha de recepción: 14 enero del 2013

Fecha de aceptación: 6 de marzo del 2013

2 Enfermera. Escuela de Enfermería, Universidad de Costa Rica Magíster en Planificación Curricular

Correo electrónico: ana.guzmanaguilar@ucr.ac.cr
} 


\section{Management curriculum: the experience of the Degree of Nursing at the University of Costa Rica ${ }^{1}$}

Ana Guzmán Aguilar ${ }^{2}$

\section{Institution: University of Costa Rica}

\section{CITED}

Guzmán, A. (abril, 2013). Management curriculum: the experience of the Degree of Nursing at the University of Costa Rica.. Rev. Actual en Costa Rica, 24, 1-14. Recuperado de:: <http://www.revenf.ucr.ac.cr/gestion.pdf> ISSN 1409-4568

\section{ABSTRAC}

We report herein the results of the third objective of the research entitled Analysis Curriculum of the Bachelor of Nursing at the University of Costa Rica. This objective is in the structure of the curriculum management plan in question. Participated in this process all teachers in the School of Nursing, teaching service, fifth year of a degree, graduates and employers in 2011-2012. We developed a mixed approach, with a parallel design. For data collection techniques were used oral and documentary instruments such as questionnaires and structured interviews to teachers, students and employers, as well as reviewing curriculum documents curriculum, The variables for the third goal were sufficient and suitability of staff, infrastructure sufficiency and adequacy and appropriateness of clinical fields. It was noted between the results of the management curriculum curriculum was broadly assessed satisfactorily in all items, leading to improvement and promote quality in the training of the professionals in nursing. We conclude that there is sufficiency and appropriateness of the teachers of the school of nursing and program of staff development is a positive action in this regard. The physical plant infrastructure still does not meet the expectations of teachers and students. Finally, the restriction on the number of students who are accepted as the current clinical field CCSS-UCR Agreement and the number of students that supports the academic unit annually makes them inadequate in clinical simulation incorporating the student achieves advantage the best learning experiences in health services.

Key words: Curriculum, Management, Nursing

\footnotetext{
${ }^{1}$ Date of receipt: January 14, 2013

Date of acceptance: March 6, 2013

${ }^{2}$ Nurse. School of Nursing, University of Costa Rica. Magister in Curriculum. E-mail. ana.guzmanaguilar@ucr.ac.cr
} 


\section{INTRODUCCIÓN}

La Gestión, según Castro (2005) es un concepto que viene de la teoría de las organizaciones, se refiere en principio a la administración de recursos; sin embargo, la evolución en la organización de las empresas ha ampliado el concepto pues existen una serie de procesos complejos que actualmente comprende la gestión y es un asunto global que desde una visión sistémica involucra la totalidad del quehacer de la institución.

La Escuela de Enfermería es una organización compleja que gestiona la enseñanza y el aprendizaje de la Enfermería, fenómenos dinámicos que no pueden verse separados de otros aspectos que están involucrados en la administración de recursos como son la planta física, el acceso a las tecnologías informáticas, la cantidad y calidad del personal docente y administrativo, los recursos financieros, entre otros. Este proceso de gestión tiene como propósito articular estos elementos para lograr el mejor resultado por lo que es preciso indagar y dar seguimiento a las necesidades e inquietudes de los actores así como a la evolución de los asuntos organizativos.

Por tanto, comprender la institución educativa como una organización, la gestión se considera como la acción colectiva que involucra a todos los actores que intervienen en el proceso de formación, tal como lo afirma Castro (2005), la participación activa de toda la comunidad educativa, debe centrarse alrededor de los aprendizajes del estudiantado.

Coherente con la posición epistemológica del plan de estudios de la Licenciatura en Enfermería de la Universidad de Costa Rica, la acción interactiva en relación al proceso formativo es una característica de la Escuela de Enfermería, donde docentes, estudiantes y administrativos son participes en la toma de decisiones respecto de las modificaciones al plan de estudios. Sin embargo, es mediante la investigación educativa que se marca el rumbo de la gestión curricular fortaleciendo la cultura de la autoevaluación y acreditación -proceso que desarrolla en la universidad en algunas unidades académicas- para hacer visibles los avances, debilidades y fortalezas de las carreras.

Consecuente con la importancia de la toma de decisiones acertadas y oportunas de la institución educativa es fundamental preparar a los docentes que asumirán a futuro el proceso directivo de la institución pues según Villela (2009) es una “ ....acción que parte del tipo de dirección de la institución y [...es...] uno de los primeros pasos para cambiar la cultura de gestión universitaria” (p. 22). Por lo que el actual modelo de gestión de la Escuela de Enfermería propicia el fortalecimiento de esta cultura.

Ahora bien, el plan de estudios representa la acción educativa de una unidad académica y un elemento concreto del currículun de la carrera, sin embargo, sin ahondar en la gran variedad de definiciones es importante comprender el significado del currículo en la gestión, como referencia se parte de la definición que propone Grundy (1994) al plantear que, 
“...el curriculum es una construcción cultural. Es decir no se trata de un concepto abstracto que tenga alguna existencia aparte de y antecedente a la experiencia humana. Es, en cambio, una forma de organizar un conjunto de prácticas educativas humanas"(s.p.)

Coincide con la cita anterior, lo que manifiesta Escudero (1999), al afirmar que el currículo es una acción deliberativa entre los participantes, (docentes, estudiantes, administrativos) que mediante consenso y argumentos sólidos logran ir construyendo y reconstruyendo la propuesta, de manera que responda en buena medida a los problemas que viven los y las profesionales en Enfermería.

De igual manera, el proceso de gestión del currículo se comprende como la gestión del conocimiento dentro de una organización para lograr la mayor calidad. Por otro lado, según López (2005), la gestión es una acción que no solo va dirigida a mejorar la información para difundirla e incorporarla a la producción de la organización o el mejoramiento individual de los y las docentes, sino que además es “...una gestión de saberes cuya finalidad es provocar cambios en las conductas de las personas en el seno de la organización para mejorarla..."'(p.26), o sea traer nuevo conocimiento, refrescar, actualizar, entre otros aspectos; esto redunda en beneficio tanto de la institución como de las personas, docentes, estudiantes, administrativos. De ahí la importancia de analizar la estructura de la gestión curricular del plan de estudios de la Licenciatura en Enfermería para conocer el alcance de este proceso en la búsqueda de la calidad.

Por lo expuesto anteriormente, el objetivo de este estudio fue analizar la gestión curricular del plan de estudios de la Licenciatura en Enfermería de la Universidad de Costa Rica.

\section{MATERIALES Y MÉTODOS}

Este estudio se enmarcó dentro de los postulados que presenta el enfoque mixto, el cual según Hernández, Fernández y Baptista. (2006), “...es un proceso que recolecta, analiza y vincula datos cuantitativos y cualitativos en un mismo estudio o una serie de investigaciones para responder a un planteamiento del problema." (p.544)

Este enfoque se fundamenta en la triangulación de métodos. El enfoque mixto va más allá de la simple recolección de datos de diferentes modos sobre el mismo fenómeno, porque implica mezclar la lógica inductiva y deductiva. Aspecto que interesó en este estudio, lograr una perspectiva del fenómeno más precisa, integral y completa. Por otro lado, incrementa la confianza en la presentación de los resultados, dado que se consideran diversas fuentes y tipos de datos, contextos o ambientes y análisis.

Respecto del diseño, se estableció la modalidad en paralelo sin combinar los datos cuantitativos con los cualitativos, se realizaron análisis separados con un único reporte. Este se refiere a la simultaneidad de los estudios cuantitativo y cualitativo, de los resultados de ambos se realizaron las interpretaciones sobre el problema planteado.

La población estuvo conformada por estudiantes de adultez sana, adultez mayor y niñez mórbida del año 2010 de la Licenciatura en Enfermería para un total de 185 estudiantes, 16 docentes de servicio con nombramiento de tiempo parcial, y a plazo fijo. 
En relación con los instrumentos y técnicas se realizó en primera instancia una revisión documental del Informe de Acreditación de la carrera de Licenciatura en Enfermería del año 2009, así como las normativas y criterios del Centro de Desarrollo Estratégico e Información en Salud y Seguridad Social (CENDEISSS) esto con la finalidad de analizar las variables suficiencia e idoneidad del recurso humano y suficiencia e idoneidad de los campos clínicos.

Seguidamente se aplicó una entrevista semiestructurada para conocer la opinión de los y las docentes en relación a esta última variable y un taller para conocer su opinión acerca de las características de los campos clínicos acreditados.

También se aplicó un cuestionario autoadministrado al estudiantado de los módulos adulto mayor, niñez mórbida y adultez sana, en donde no hay intermediarios y las respuestas las marcan los y las participantes (Hernández, et al., 2010). El instrumento tuvo como objetivo conocer la opinión de los y las encuestadas acerca de la suficiencia e idoneidad de los campos clínicos, el número y equipamiento de salas de simulación y la condición y cantidad del equipo informático disponible, así como la idoneidad y sufíciencia del Centro de Atención y Tecnología para Enfermería.

\section{Consideraciones éticas}

Esta investigación fue desarrollada bajo los siguientes principios éticos. El principio de autonomía se respetó al aclarar a cada persona sus posibilidades de aceptar o negar su participación voluntaria en el estudio o la posibilidad de solicitar en cualquier momento ser excluido en la investigación. Se garantizó la beneficencia y no maleficencia desde el objetivo general de la investigación, pues la unidad académica podrá tomar acciones de mejoramiento, en busca de la excelencia en la formación de profesionales. Además, a cada informante se le explicó la naturaleza y finalidad de la investigación y se mantuvo la privacidad y anonimato en todo momento.

\section{RESULTADOS}

\section{Suficiencia e idoneidad del recurso humano}

Según el documento de avance del proceso de reacreditación de la Escuela de Enfermería -en relación al criterio de suficiencia- a partir del 2010 a la fecha ha brindado mejores condiciones laborales para el personal docente. Lo anterior se evidencia por el descongelamiento de ocho plazas docentes y la adjudicación de dos tiempos completos en docencia que permiten una adecuada asignación de funciones, aspecto que se ve reflejado en la mejor distribución de las cargas académicas, tiempo real para realizar investigación y tiempo real para impartir tutorías en los trabajos finales de graduación.

En cuanto al criterio de idoneidad se encontró que para el primer semestre del 2012 se contó con 27 docentes en propiedad y 35 en condición de interinos. El grado académico de 22 profesoras en propiedad es de maestría, tres con grado de doctorado y una con licenciatura. El personal docente interino por su parte, 15 tienen el grado de magíster y 20 de licenciatura. Se destaca que este personal interino varía debido al número de estudiantes matriculados en los módulos y en el curso de Gestión del Cuidado, que demandan un mayor número de campos clínicos por semestre y por ende el número de contrataciones interinas. 
Según el plan de desarrollo institucional 2008-2012 de la Universidad de Costa Rica, hay tres ejes del quehacer universitario (docencia, acción social e investigación) sobre los que deben trabajar todos y todas los universitarios, pero en especial el cuerpo docente. En ese sentido, el profesorado de la Escuela de Enfermería ha incursionado significativamente en el eje de la investigación, se destacan 18 proyectos inscritos en la Vicerrectoría de Investigación, lo que involucra un número importante de docentes en estos procesos, de la misma manera en el eje de Acción Social aumentaron el número de proyectos.

Otros aspectos que refuerzan la idoneidad del profesorado es la reserva de cinco plazas docentes para realizar estudios doctorales en universidades extranjeras, en la actualidad estas plazas están adjudicadas a profesores y profesoras que están realizando sus estudios en la Universidad de Concepción en Chile, Universidad de Miami, Universidad de Virginia en los Estados Unidos de Norteamérica y en la Universidad de Florianópolis, Brasil.

El proceso de gestión para mejorar las condiciones del cuerpo docente y la suficencia del mismo implicó crear otras estrategias como son el descongelamiento de plazas, asignación de tiempo docente para nombramiento continuo a interinos, reserva de plazas docentes para estudios doctorales, así como, una mejor distribución de las cargas para atender otras áreas sustantivas del quehacer docente. ${ }^{1}$

\section{Suficiencia de la Infraestructura}

El edificio de la Escuela de Enfermería está ubicado en la finca 2 de la Ciudad de la investigación, Sabanilla de Montes de Oca y conformado por dos edificios, sin embargo se hace insuficiente dicho espacio para cubrir diversas necesidades, las cuales se anotan a continuación:

1. Albergar a 415 estudiantes del programa de Licenciatura en Enfermería (Acreditación, 2012) y 150 estudiantes del programa de posgrado en Ciencias de Enfermería el cual cuenta con siete maestrías.

2. Pocas oficinas para el número de docentes que laboran en la Escuela de Enfermería tanto en grado como en posgrado. (Plan de Gestión y Atención de Emergencias 2011)

3. El crecimiento de la Escuela de enfermería en las diferentes áreas sustantivas del quehacer universitario genera mayores necesidades tanto de contar con más personal administrativo y misceláneo lo que conlleva a requerir mayor espacio físico.

4. El espacio con que cuenta la Asociación de Estudiantes es limitado.

\footnotetext{
${ }^{1}$ En cuanto a la idoneidad comparada con los criterios establecidos por el SINAES, podemos afirmar que la planilla docente posee y supera ampliamente dichos estándares.
} 
5. Poco espacio físico para actividades docentes entre ellas la atención a estudiantes, reuniones de comisiones. Además, las oficinas del segundo y tercer piso, según el Plan de Gestión y Atención de Emergencias (2011) son insuficientes y generan hacinamiento.

6. La disponibilidad de aulas es insuficiente para cubrir las necesidades de la población estudiantil del grado y posgrado, debido a que el edificio tiene sólo 3 aulas: dos de ellas con una capacidad para 40 estudiantes y la otra para 25. El auditorio está ubicado en el tercer piso con capacidad para 60 personas.

\section{Suficiencia e idoneidad de Campos Clínicos}

La distribución de campos clínicos en los módulos y el curso de Gerencia del Cuidado están organizados en dos grandes escenarios, el primero de ellos es la parte asistencial constituida por hospitales, clínicas, centros y áreas de salud, estos pertenecen a la CCSS y son administrados por el Centro de Desarrollo Estratégico e Información en Salud y Seguridad Social (CENDEISSS). El segundo de los escenarios corresponde a instituciones educativas públicas pertenecientes al Ministerio de Educación Pública de Costa Rica, así como organizaciones gubernamentales y no gubernamentales que atienden a diversos grupos etarios y poblaciones en condición de vulnerabilidad o riesgo, dentro de ellos: instituciones de estancia para el tratamiento y rehabilitación de las adicciones, albergues del Patronato Nacional de la Infancia, centros diurnos y hogares de ancianos.

Es importante destacar que la suficiencia e idoneidad de los campos clínicos seleccionados por la Escuela de Enfermería para el primer escenario mencionado en el párrafo anterior, depende de la acreditación que realiza el CENDEISSS de los mismos. Este cuenta con criterios mínimos para determinar la idoneidad de escenarios clínicos, dentro de estos se tiene: “...número de pacientes, giro cama, servicios aptos para la docencia según carrera y curso, número de funcionarios, experiencia del docente, número de experiencia por docente, objetivos de la práctica, infraestructura, recursos materiales para la docencia." (Reglamento de la actividad clínica docente, Caja Costarricense del Seguro Social, 2010, p.2)

Respecto de lo anterior, un docente manifestó, "Suficientes no, pienso que pueden haber más campos clínicos para disminuir la cantidad de estudiantes por campo clínico. Idóneos sí unos más que otros dependiendo del personal..." (sujeto 4)

Otro docente expresó "En general son muy buenos, a veces un poco de dificultad. La parte burocrática nos afecta mucho. Si vienen grupos muy grandes no tenemos donde ubicarlos.” (sujeto 3)

Las opiniones anteriores evidencian que los y las docentes (9) consideran que los campos clínicos son idóneos, pero no suficientes, debido a la demanda cambiante de la población estudiantil. Para tener una mayor comprensión de esta variable se analizó las opiniones del estudiantado sobre la suficiencia e idoneidad de los campos clínicos.

Cabe destacar que en casi todas las respuestas de las personas participantes se ubicaron en los rubros de muy de acuerdo y de acuerdo respecto a su opinión sobre la suficiencia para el logro de los objetivos en los diferentes módulos. Los y las estudiantes opinaron en un 57,3\%, que fueron suficientes y un $82,7 \%$ que son 
idóneos, respuestas que coinciden con lo expresado por las (os) docentes, por ejemplo uno de ellos dijo: “yo pienso que si son los idóneos -los estudiantes pueden realmente planear, implementar, diagnosticar, evaluar. Todo eso lo puede hacer el estudiante.". (Sujeto 7)

En relación con la opinión de los (as) egresados sobre la variable de idoneidad de los campos clínicos por módulos se obtuvo para una muestra de 43 participantes que el módulo con mayor número de respuesta favorables (30), fue el de la Niñez Sana, seguido por el Módulo de la Niñez Mórbida con 23 opiniones, el Módulo de la Adolescencia ocupó un tercer lugar con 29 respuestas y finalmente, el Módulo de la Adultez Sana y la Adultez Mórbida con 25 y 24 opiniones favorables respectivamente.

En cuanto a la variable suficiencia de los espacios de práctica utilizados para el logro de los objetivos de los módulos, tuvo un comportamiento similar a la variable de idoneidad entre los y las egresadas; de aquí que de nuevo el módulo que agrupó mayor número de respuestas favorables es el de la Niñez Sana con 25 opiniones igual que el Adulto mayor, seguido por la Niñez mórbida con 23 respuestas, los demás módulos rondan entre 18 y 23 opiniones.

La suficiencia del tiempo asignado en los espacios de práctica para el logro de los objetivos de los módulos, las opiniones favorables rondaron entre 26 y 17 respuestas. Sin embargo, se debe enfatizar que, aún cuando el grupo que considera que el tiempo no fue suficiente, es menor, estas opiniones se ubican en su mayoría en el curso de Gestión del cuidado (12), los Módulos de la Adultez Sana (13) y Mórbida (15), los mismos que en opinión de las personas egresadas no fueron idóneos para el logro de los objetivos y en los que se tuvo que modificar la práctica pues no respondían a los objetivos planteados.

Los y las docentes encuestadas apuntan dificultades que se experimentan en los campos clínicos, que inciden directamente con el aprovechamiento del tiempo de estancia en los mismo, el siguiente ejemplo lo resume: "No son suficientes, se han tenido muchos problemas, y no se aprovechan del todo el campo clínico, llegan tarde, se van antes, etc. Al haber necesidad de campos, se tienen campos que no cumplen con requisitos. En ocasiones se tienen buenos campos pero no se pueden aprovechar, ejemplo en el Hospital de Niños no se pueden tocar a los niños". (sujeto 13).

El impacto de lo mencionado hasta aquí se puede ver reflejado en la opinión de los y las egresadas quienes expresan que el tiempo asignado fue poco, por lo que no se logra aprovechar todas las experiencias clínicas, opinando que el conocimiento teórico que se ofrece en el plan de estudios es bueno ( 23 respuestas) pero no así la práctica, que es considerada poca.

\section{Acceso al centro de simulación y equipo de cómputo.}

Otro aspecto investigado que es de gran importancia por las implicaciones éticas y prácticas que posee es el Centro de Simulación Clínica en Salud (CESISA). Se indagó sobre la suficiencia en cantidad y calidad del equipamiento de la sala de simulación para el logro de los objetivos clínicos. Se evidencia, un porcentaje de respuestas de acuerdo y muy de acuerdo de $46,5 \%$ y en desacuerdo y muy en desacuerdo el 53,5\%. Finalmente, en lo que respecta al equipo de la sala y equipo de cómputo las opiniones evidencian suficiencia en cantidad y calidad para un porcentaje de acuerdo y muy de acuerdo de $92,5 \%$ y $88,6 \%$. 
Coincidente con la información aportada por los y las estudiantes, está la opinión del personal docente, para quienes el equipo de cómputo y el CESISA están dando un gran aporte a la formación principalmente en el campo del cuidado asistencial, así lo expresa una docente "Si claro suple muchas necesidades que en el campo clínico se han visto limitadas. Da confianza al estudiante, les da una pequeña idea de cómo es la enfermería "(sujeto 9) y reiteran sus opiniones en cuanto a aumentar los servicios que se brindan dadas las necesidades cada vez mayores de la unidad académica en cuanto a la implementación de tecnologías en la enseñanza, esto se plasma en los siguientes comentarios: "Se requiere ampliación en las salas debido a que la población que tenemos en nuestra escuela es muy amplia" (sujeto 5), "No es suficiente, debemos de luchar por lo que realmente debe ser"(sujeto 8), "Creo que puede ampliarse hemos visto que no es suficiente -- Están bien” (sujeto 4), "Está bien pero podrían ampliarse. Podrían ampliarse tanto los equipos como los horarios"(sujeto 10).

Por otra parte, al analizar los registros del CESISA (oficio EECESISA-1016-06-2012, enviado a la Comisión de Autoevalución), es evidente la utilización que realiza el estudiantado de las actividades de simulación programada por el centro, las cuales se brindan como actividades extracurriculares, lo cual muestra la aceptación de la población estudiantil, se evidencia en los registros del I semestre del 2012 que se atendieron 330 estudiantes, para lo cual el CESISA invirtió 838 horas, esto ha generado un crecimiento importante que puede ser medido en términos de la adquisición de 16 nuevos modelos y equipos, que redunda en una mejora de la oferta de servicios que brinda a la comunidad docente y estudiantil.

\section{DISCUSION}

Al considerar la suficiencia, idoneidad y calidad de los y las docentes desde su formación académica y experiencia profesional no se debe dejar de lado la educación permanente, entendida como,

un proceso de actualización que le posibilita realizar su práctica pedagógica y

profesional de una manera significativa, pertinente y adecuada a los contextos sociales en que se inscribe y a las poblaciones que atiende. Es así como dicha formación se presenta articulada al ejercicio mismo de la práctica pedagógica y a formas de entenderla e inscribirla en los contextos sociales donde se realiza. Y en esta misma forma se concibe al docente como un profesional capacitado (idóneo) para reconstruir el conocimiento pedagógico, a partir de la experiencia a que se enfrenta cotidianamente. (Carmago, et al. 2004, p.81)

Por la importancia que posee la educación permanente esta inscrita como una política tanto institucional como de la unidad académica, la cual esta expresada en la planificación anual de la gestión actual de la dirección de la Escuela de Enfermería, denominado "Programa de Desarrollo del Recurso Humano Docente", el cual esta organizado en actividades de capacitación en diversas áreas del quehacer docente como didáctica y evaluación de los aprendizajes y actualización en diversas temáticas, que se ofrecen semestralmente. (Plan de gestión académica de la Escuela de Enfermería de la Universidad de Costa Rica, periodo 2010- 2014)

La idoneidad del profesorado en los aspectos pedagógicos es fundamental para lograr que el estudiantado en situaciones diversas pueda utilizar esos conocimientos en la resolución de problemas de su futura práctica profesional, tal como lo afirma Salazar (2012) cuando menciona que, 


\section{Revista Electrónica Enfermeria Actual en costa Rica}

el manejo de contenidos para la enseñanza se asocia con el conocimiento que tiene el docente sobre los saberes disciplinares que enseña, el cual se convierte en un elemento fundamental pues no es posible pensar lo pedagógico en el vacío; no obstante, este conocimiento no se puede entender sino es en relación con lo pedagógico. Tener manejo de los saberes disciplinares que se enseñan, requiere el reconocimiento de la estructura de conceptos y procedimientos que tiene el contenido. Así, el docente está facultado para identificar los principales obstáculos que existen para aprender y conocer esos contenidos.( p.30)

Por lo que las acciones emprendidas por la dirección de la unidad académica en este sentido son valiosas ya que la docencia en Enfermería requiere de diversas estrategias didácticas algunas muy conductuales porque hay procedimientos que de acuerdo a las principios de asepsia no pueden variarse en el riguroso seguimiento de pasos, como la curación quirúrgica, no así otras actividades donde el ingenio y la creatividad son la clave, como la administración de los escasos recursos materiales y humanos en servicios con plétora de usuarios (as) a los que hay que atender en sus necesidades prioritarias, son prácticas que debe tener el estudiante y que el docente debe planificar cuidadosamente para que sean realmente experiencias de aprendizaje.

Así mismo, la unidad académica a determinado que el modelo pedagógico a seguir es el constructivismo, por tanto se encuentra coherencia con la definición que también proporciona López ( 2005), cuando afirma que "“...el enfoque constructivista toma en cuenta la diversidad de las personas, considera que el aprendizaje es para dar sentido a su existencia y que todo el aprendizaje se centra en el contexto funcional y social." ( p. 127)

Otro elemento importante en la calidad de la panificación y realización de las acciones en la institución depende de las competencias que tengan los involucrados en los procesos, principalmente quienes diseñan la formación, pues son quienes determinan qué y cómo se aprende la Enfermería, así que este proceso exige un dominio significativo de aspectos relacionados con las funciones que desempeñan, por lo que se requiere también identificar necesidades, escuchar opiniones y favorecer una actitud propicia para el aprendizaje.

Igualmente “...corresponde atender las expectativas personales y profesionales de quienes son los gestores directos de los procesos" (López, 2005, p.166), pues existen factores que son claves para lograr un buen ambiente organizativo y alcanzar una mayor motivación como son: las oportunidades de promoción profesional, el sentimiento de utilidad del trabajo realizado, el grado de conocimiento acerca de la política, nivel de responsabilidad y autonomía de las personas para desarrollar su trabajo, la comunicación interna, el grado de participación de las y los docentes para definir los objetivos del trabajo, las relaciones en la unidad de trabajo, el nivel retributivo, la confianza mostrada por el director (a) hacia el profesorado, entre otras.

El diagnóstico del estado de la organización, junto con el establecimiento de indicadores y objetivos a lograr por la institución nos lleva a seguir un plan de acción que contemple las necesidades antes mencionadas y sentidas por el cuerpo docente, en este sentido la dirección de la unidad académica ha implementado el plan de Desarrollo de Personal Docente y Administrativo del 2011 al 2014, con la intención de que las acciones a realizar sean consecuentes con las demandas de los formadores y colaboradores del proceso formativo. 
Como se mencionó anteriormente un criterio de calidad en la formación de los profesionales de Enfermería lo constituye la planta física de la institución, y la investigación confirmó las debilidades que en este aspecto presenta la unidad académica para satisfacer las necesidades de docentes y estudiantes, sin embargo, para Duarte (2005) el ambiente educativo se refiere tanto al medio físico que rodea a la institución como a las interacciones que se producen en este medio, la disposición espacial de las estructuras, las relaciones entre estas, así como las pautas de comportamiento y el tipo de relaciones que mantienen las personas entre si y con los objetos, los criterios que prevalecen y las actividades que ahí se realizan.

Por tanto, no deben valorarse solamente la cantidad y calidad de las estructuras si no también considerar aspectos como "la calidez" del lugar y las acciones que se realizan para favorecer un espacio de convivencia pacífica, aspectos que también se toman en cuenta en la planificación de actividades para estudiantes y docentes como celebración de cumpleaños, y festividades que propician este clima de buenas relaciones, sin dejar de lado la organización del espacio y la ambientación de áreas agradables para la convivencia.

Respecto de los campos clínicos, se sabe que la formación de un buen profesional de enfermería depende en mucho del tipo de experiencias de aprendizaje que se le ofrezcan a lo largo de todo su plan de estudios, por esto es que la selección de los campos clínicos es una de las principales labores de la gestión académica, es en la práctica clínica donde se establece la relación con las personas que requieren del cuidado y se concreta el acto de cuidar. Por lo que se debe recurrir a los saberes aprendidos para articular conceptos teóricos con la búsqueda de soluciones a situaciones prácticas concretas, reales y donde la capacidad para la toma de decisiones se va convirtiendo en una práctica cotidiana. Se requiere del juicio crítico para determinar las mejores alternativas del cuidado, planificar acciones de promoción, prevención, cuidado directo y otras actividades propias de la Enfermería que determinan la calidad y la efectividad del quehacer profesional, así como condiciones estructurales y de equipo tecnológico adecuado para tal fin. (Cid y Sanhueza, 2006)

Se destaca que la Escuela de Enfermería -cuando selecciona los campos clínicos para desarrollar sus actividades formativas- toma en cuenta los criterios que establece el CENDEISSS para la acreditación de los mismos; sin embargo, existen factores que influyen directamente sobre la suficiencia e idoneidad de éstos, como son el número de estudiantes permitidos por el CENDEISSS y el número de personas matriculadas en los módulos o en el curso de Gestión del cuidado, de tal manera que se tiene que acudir a la estrategia de disminuir el tiempo de estancia en el lugar de práctica y por ende, el número de experiencia al que tiene acceso el estudiantado.

Una buena selección de campos clínicos implica por tanto, conocer los espacios laborales, y tener capacidad de negociación con distintas entidades públicas y privadas que cuenten con servicios de calidad y cumplan con las expectativas de la institución que en muchas ocasiones supera las posibilidades, por lo que también se realizan acciones paralelas para maximizar estas experiencias como el fortalecimiento de la simulación clínica.

Lo anterior es otro aspecto importante de analizar pues la simulación clínica, es una práctica que a nivel nacional e internacional cada vez cobra mayor relevancia en los programas de enfermería, por los beneficios que tiene en el proceso de enseñanza y aprendizaje ya que permite optimizar la asistencia y 
maximizar la seguridad clínica del usuario (a), minimizando el error y acercando al estudiantado a situaciones reales de la práctica clínica diaria para identificar las prioridades y posibles errores.

La simulación clínica es una experiencia de aprendizaje que actualmente ha incursionado fuertemente en la gestión del plan del estudio y su objetivo fundamental es lograr el máximo provecho de las oportunidades de aprendizaje en el campo clínico, además, acelera la adquisición de habilidades y conocimientos en un ambiente seguro sin riesgos para las personas que reciben el cuidado.

La idoneidad y suficiencia de los campos clínicos tal como lo evidenció la investigación, se ha visto afectada principalmente por la cantidad de estudiantes aceptados por campo clínico según el Convenio CCSSUCR del 2011, pues solamente se aceptan cuatro o cinco estudiantes por grupo, en un módulo de 10 o 12 grupos hace que la simulación de técnicas y procedimientos sea actualmente una importante alternativa para lograr el aprendizaje de Enfermería en ambientes controlados y con escenarios previamente diseñados, donde existe la posibilidad de repetir las acciones y sobre todo de analizar individual y conjuntamente los cuidados realizados.

Es la oportunidad que tienen docentes y estudiantes de analizar situaciones, ser autocráticos pues es un aprendizaje interactivo con devolución inmediata del resultado de la intervención.

Actualmente el reto que enfrenta la unidad académica está en articular estas acciones al plan de estudios de manera que se aplique en todo el proceso formativo, de todos los y las estudiantes, en distintos niveles de complejidad según avanza en la carrera, tratando de superar como se vio anteriormente las limitaciones en el espacio físico.

\section{CONCLUSIONES}

Existe suficiencia e idoneidad de los y las docentes de la escuela de Enfermería y el programa de desarrollo del personal docente es una acción positiva en este sentido.

La Infraestructura de la planta física aun no cumple con las expectativas de docentes y estudiantes.

Por último, la restricción en el número de estudiantes que se aceptan por campo clínico según el actual Convenio CCSS-UCR y la cantidad de estudiantes que admite la unidad académica anualmente hace que sean insuficientes por lo que al incorporar la simulación clínica el y la estudiante logran aprovechar al máximo las experiencias de aprendizaje en los servicios de salud. 


\section{Agradecimientos}

Se agradece profundamente el apoyo que se brindó para concluir con éxito esta

Investigación a :

M.Sc Antonieta Calvo Rodríguez, Directora colaboradora hasta Julio 2010.

M.Sc. Ligia Murillo Castro, Directora colaboradora, Julio 2010 a la fecha.

Comisión de Reconocimiento, Credenciales y Curriculum, 2006 a Febrero 2012

Dra. Ligia Rojas Valenciano, Coordinadora

M.Sc Ana Guzmán Aguilar, Miembro

M.Sc Viriam Leiva Díaz, Miembro

M.Sc. Mabel Granados Hernández, Miembro

M.Sc Rebeca Gómez, Miembro

M.Sc Derby Muñoz, Miembro

\section{Comisión de Reconocimiento, Credenciales y Curriculum, Febrero 2012 a la fecha}

M.Sc Rebeca Gómez, Coordinadora

M.Sc. Mabel Granados Hernández, Miembro

M.Sc Laura López, Miembro

M.Sc Dennis Carrillo, Miembro

M.Sc. Alba Carranza, Miembro

M.Sc Elena Mora Escalante

\section{Otros colaboradores}

Docentes de la Escuela de Enfermería de la Universidad de Costa Rica

Estudiantes de Enfermería de la Universidad de Costa Rica de la promoción del 2011.

\section{REFERENCIAS}

Castro F. (2005). Gestión curricular: una nueva mirada sobre el curriculum y la institucion educativa. Horizontes Educacionales, 13-25.

Recuperado de: http://redalyc.uaemex.mx/redalyc/pdf/979/97917573002.pdf

Carmago, M., Calvo, G., Franco, C., Vergara, M., Londoño, S., Zapata, F. y Garavito, C. (2004). Las necesidades de formación permanente del docente. Rev Educación y Educadores, 7 Recuperado de: http://redalyc.uaemex.mx/pdf/834/83400708.pdf

Caja Costarricense del Seguro Social (2010) Reglamento de la actividad clínica docente. Convenio CCSS y UCR para el uso de campos clínicos. Gaceta N 112 del 10 de junio del 2010.

Centro de Simulación Clínica en salud (2012). Informe para la Comisión de Acreditación Oficio EECESISA 1016-06-2012 Escuela de Enfermería Universidad de Costa Rica. 
Cid, P. y Sanhueza, O. (2006) Acreditación de campos clínicos de enfermería. Rev. Cubana Enfermería, 22 (4) Recuperado de:<http://scielo.sld.cu/scielo.php?script=sci_arttext\&pid=S0864-

03192006000400010\&lng=es\&nrm=iso $>$.

SINAES (2009). Manual de Acreditación Oficial de Carreras de Grado del Sistema Nacional de Acreditación de la Educación Superior. Recuperado de:

http://www.sinaes.ac.cr/manual_guias/manual_oficial_acreditacion_vf_feb2010.pdf

Duarte, J.( 2005) Ambientes de Aprendizaje una aproximación conceptual

Revista Iberoamericana de Educación Recuperado de: http://www.rieoei.org/deloslectores/524Duarte.PDF

Escudero, JM.,(1999) Diseño, desarrollo e innovación del curriculum. España Edit. Síntesis educación.

Escuela de Enfermería (1998) Estudio para la modificación del plan de estudios de la carrera de Licenciatura en enfermería San Pedro, Universidad de Costa Rica. Tomo I

Escuela de Enfermería (2012) Informe de Compromiso de Mejora año II Comisión de Autoevaluacion y Acreditación . Universidad de Costa Rica.

Escuela de Enfermería (2009) Informe para la Acreditación de la carrera de Licenciatura en Enfermería. Comisión de Autoevaluacion y Acreditación. San Pedro Universidad de Costa Rica .

Grundy, S. (1994). Producto o praxis del currículo. Segunda edición. Madrid, España: Ediciones Morata. S.L.

Hernández, R., Fernández, C. y Baptista, P. (2010). Metodología de la investigación. México: Editorial McGraw-Hill.

López, J. (2005) Planificar la formación con calidad. España: Graficas Muriel.S.A.

Salazar, S. F. (2012) El conocimiento pedagógico del contenido como modelo de mediación docente [multimedio] / - 1ª . ed. -- San José, C.R. : Coordinación Educativa y Cultural.

Villela, A. ( 2009)Gestión en Educación Superior: Funciones, capacidades y necesidades directivas. Recuperado de: http://www.revistaakademeia.cl/wp/wp-content/uploads/2011/09/Gestion-en-educacion-superior.pdf 